\title{
SERS Properties of Gold Nanorods at Resonance with Molecular, Transverse, and Longitudinal Plasmon Excitations
}

\author{
Ida Ros • Tiziana Placido • Vincenzo Amendola • Chiara Marinzi • Norberto Manfredi • \\ Roberto Comparelli • Marinella Striccoli • Angela Agostiano - Alessandro Abbotto • \\ Danilo Pedron • Roberto Pilot • Renato Bozio
}

Received: 27 October 2013 / Accepted: 22 January 2014 / Published online: 7 March 2014

(C) The Author(s) 2014. This article is published with open access at Springerlink.com

\begin{abstract}
The amplification of Raman signals of the heteroaromatic cation 1-(N-methylpyrid-4-yl)-2-(Nmethylpyrrol-2-yl)ethylene (PEP+)) bound to Au nanorods (NRs) was investigated at different excitation wavelengths to study the effect of the laser resonance with the absorption band of the PEP+ moiety and with the two plasmon oscillation modes of the NR. Two different PEP+ derivatives, differing in the length of the alkyl chain bearing the anchoring group, were used as target molecules. Raman spectra obtained exciting at 514 or at $785 \mathrm{~nm}$ (i.e., exciting the transverse or the longitudinal plasmon band) present a higher intensity than that at $488 \mathrm{~nm}$ suggesting a higher Raman amplification when the laser excitation wavelength is resonant with one of the two plasmon modes. Moreover, considering results of Discrete Dipole Approximation (DDA) calculations of the local field
\end{abstract}

I. Ros $(\square) \cdot$ V. Amendola $\cdot$ D. Pedron $\cdot$ R. Bozio $(\square)$

Department of Chemical Sciences, University of Padova, Via

Marzolo 1, 35131 Padova, Italy

e-mail: ida.ros@libero.it

e-mail: renato.bozio@unipd.it

I. Ros $\cdot$ R. Bozio

INSTM, University of Padova, Via Marzolo 1, 35131 Padova, Italy

T. Placido $\cdot$ A. Agostiano

Department of Chemistry, University of Bari, Via Orabona 4, Bari 70126, Italy

C. Marinzi $\cdot$ N. Manfredi $\cdot$ A. Abbotto

Department of Materials Science and INSTM, University of

Milano-Bicocca, Via Cozzi 53, 20125 Milano, Italy

R. Comparelli $\cdot$ M. Striccoli $\cdot$ A. Agostiano

CNR-IPCF Division of Bari, c/o Department of Chemistry,

University of Bari, Via Orabona 4, Bari 70126, Italy

R. Pilot

Department of Chemical Sciences, University of Padova and

INSTM, Via Marzolo 1, 35131 Padova, Italy generated at the NR surface when either the transverse or the longitudinal plasmon modes are excited, we deduced that the resonance condition of the 514-nm laser excitation with the absorption band of the dye strongly contributes to the amplification of the Raman signal.

Keywords Gold nanorods · Localized surface plasmon resonance $\cdot$ SERS $\cdot$ Nonlinear optics $\cdot$ Push-pull molecule

\section{Introduction}

Raman spectroscopy, owing to its ability to recognize typical molecular fingerprints, provides a unique approach for solving analytical problems [1]. However, Raman scattering has an extremely small cross section, typically $10^{-30}$ to $10^{-25} \mathrm{~cm}^{2}$, which prevents the use of this technique as a method for ultrasensitive trace detection. Raman scattering literally appears in a new light when it takes place in the local optical field of metal nanostructures [2]. These systems, thanks to the ability of sustaining strong localized surface plasmon resonances (LSPR), provide the key effect for the observation of enhanced Raman signals from molecules attached to them (Surface Enhanced Raman Spectroscopy-SERS). The enhancement depends on the type of metal and on the nanostructure features and it is particularly strong when both laser and scattered field are in resonance with the surface plasmons [3].

Several examples of nanostructures of different shape, metal and, possibly, assembled in ordered or random arrangements are reported in the literature, resulting in electromagnetic SERS enhancement factors up to $10^{8}[1,4-10]$. For example, rod-shaped metal nanoparticles possess two plasmon resonances, a transverse mode perpendicular to the long axis of the rod and a longitudinal mode parallel to the long rod axis [11]. The latter depends linearly on the aspect ratio, i.e., the length divided by the width of the nanorods (NRs), and it 
is widely tunable in the visible and in the near infrared region of the spectrum. In addition, both plasmon resonances depend on the aggregation state of the nanoparticles and on the refractive index of the surrounding medium. The possibility to tune the resonance wavelength can be exploited to amplify both laser and scattered field in SERS measurements at the desired wavelength.

Despite the attractive characteristics of metallic NRs as SERS substrates, only a few reports exist for SERS on NRs where the Raman excitation occurs at a wavelength that overlaps with NRs plasmon resonance [12-18]. To our knowledge, only Orendorff et al. [15] and Guo et al. [17] conducted a shape-dependent SERS study in dilute, not aggregated, colloids of silver and gold NRs, concluding that overlapping the excitation line with the longitudinal SPR contributes an additional enhancement by a factor of $10-10^{2}$.

In this work, we investigate the amplification of the Raman signals of the heteroaromatic cation 1-(Nmethylpyrid-4-yl)-2-( $N$-methylpyrrol-2-yl)ethylene (PEP+) bound to $\mathrm{Au}$ NRs. The dipolar, positively charged dye PEP+ (Fig. 1) is a push-pull molecule composed by a $\pi$ deficient (pyridinium ion) as the acceptor group (A) and a $\pi$-excessive (pyrrole) heterocycle as the donor group (D) [19]. PEP+ and its derivatives present remarkable resonant and non-resonant nonlinear optical properties, such as two-photon absorption and second harmonic generation [20-22]. In particular, we have thoroughly investigated the linear and non-linear optical properties of this class of pushpull systems for application in optical limiting and upconverted lasing devices [23-25]. To improve the hyperpolarizability, and in so doing the nonlinear optical properties, chemists have been modifying the electron affinity of the acceptor and the ionization potential of the donor groups by selecting more or less polar substituents. The amplified local field at the surface of metal nanoparticles is a further possible means of enhancing the effective nonlinear optical responses. A preliminary investigation of the SERS properties of this type of dye opens the way to evaluating the effectiveness of this enhancement since both SERS and two-photon absorption exhibit a similar dependence on the fourth power of the local electric field.

In order to graft the PEP+ ion on Au NRs, we have synthesized acetylthio terminated PEP+ chromophores spaced by two linear alkyl chains of different length, containing $3(\mathbf{P E P}+\mathbf{C 3 S A c})$ or $12(\mathbf{P E P}+\mathbf{C 1 2 S A c})$ carbon atoms (Fig. 1).

The basic idea is to control the intensity of the Raman signals as a function of the distance between the dye and the metallic surface of the metal nanostructure and to study the effect of the resonance of laser wavelengths with the absorption band of PEP+ moiety (molecular resonance) and with the two plasmon modes (plasmonic resonances).

The Raman characterization of NRs functionalized with $\mathbf{P E P}+\mathbf{C} 3 \mathrm{SH}$ and $\mathbf{P E P}+\mathbf{C 1 2 S H}$, both deposited on glass substrates and in solution, is realized using different laser excitation lines. The 488-nm and the 514-nm laser excitation lines are in near resonance with the absorption band of the molecules, yielding surface enhanced resonance raman scattering (SERRS), whereas the 785-nm laser excitation line is offresonant with molecular excitations. On the other hand, the 514-nm and the 785-nm lines are resonant with the transverse and longitudinal modes of Au NRs, respectively, suggesting a SERS-type enhancement of the Raman signals.

4-Mercaptopyridine bound to NRs is used to calculate the SERS enhancement factor (EF) for the transverse and longitudinal plasmon band excitations. Without possible interference with molecular resonances, the measured EFs provide an experimental probe of the local field. The results turn out to be in fair agreement with the theoretical values obtained by the Discrete Dipole Approximation (DDA) calculation of the local electromagnetic field intensity around a single NR.

\section{Results and Discussion}

Optical and Morphological Characterization of Bare Au NRs

CTAB-stabilized Au NRs prepared by seed mediated growth method exhibited a transverse and longitudinal plasmon absorption bands at 520 and $670 \mathrm{~nm}$, respectively. The normalized extinction spectrum of CTAB-stabilized Au NRs in water is shown in Fig. 2a, the dotted line corresponds to the orientationally averaged extinction spectrum obtained via the DDA method. The average size of NRs was worked out by averaging more than hundred particles in TEM images (a typical one is reported in Fig. 2b). The average dimensions turn out to be
Fig. 1 Molecular structures of parent dipolar species PEP+ and its $\omega$-acetylthioalkyl derivatives PEP+C3SAc and PEP+ C12SAc

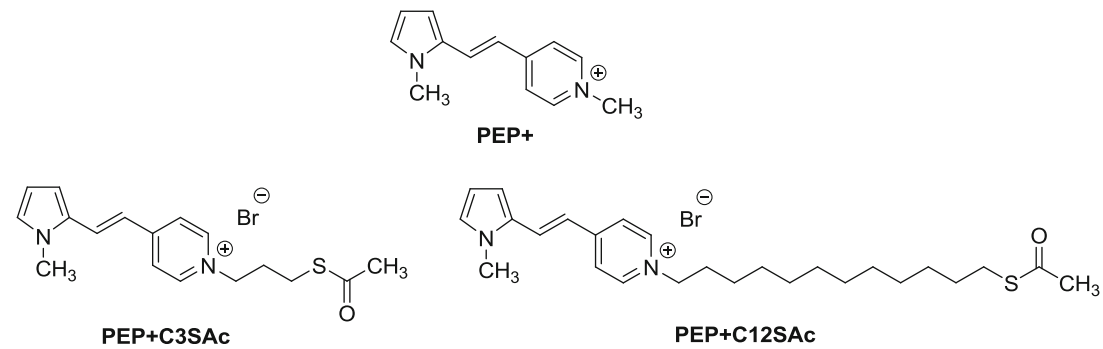


Fig. 2 Extinction spectrum (a) and relative TEM image (b) of water soluble $\mathrm{Au}$ NRs prepared by seed mediated method. The dotted line corresponds to the extinction spectrum calculated by the DDA method
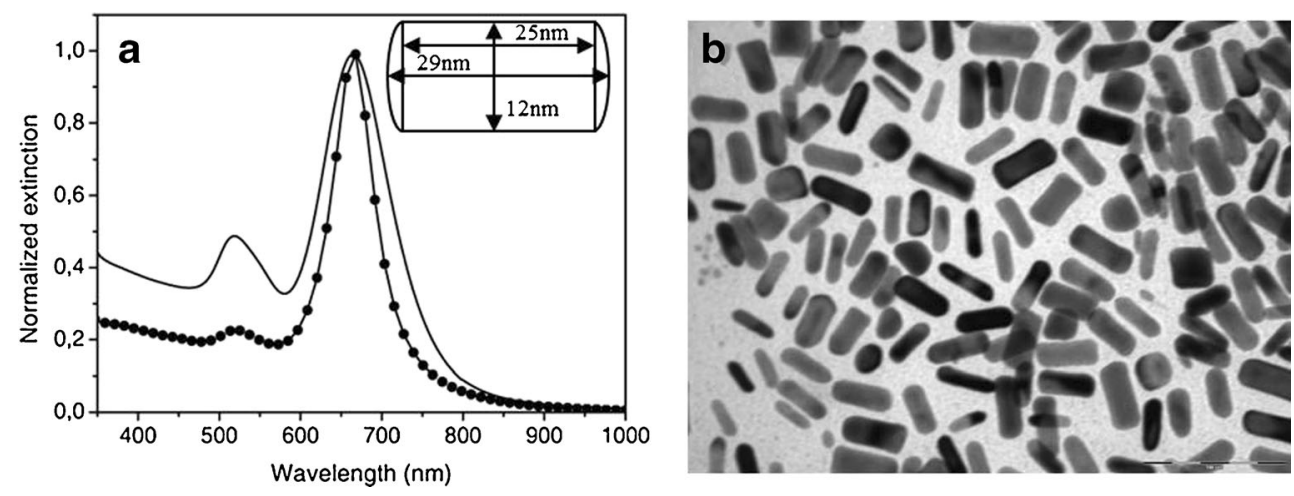

$29 \pm 3 \mathrm{~nm}$ in length and $12 \pm 2 \mathrm{~nm}$ in diameter, thus the aspect ratio (length to diameter) is approximately 2.4.

Both experimental and theoretical spectra in Fig. 2 have been normalized to the maximum extinction, that is the peak intensity of the longitudinal plasmon.

Calculated and experimental spectra differ in two features, namely: (i) the different intensity ratio between longitudinal and transverse plasmon and (ii) the larger spectra width of the longitudinal plasmon resonance in the experimental spectrum. Looking at the TEM image of our sample in Fig. 2b, where over 100 particles are imaged, just few roundish/cubic NPs are evident (around $8 \%$ ), therefore we find unlikely that (i) arises from the presence of spherical particles whose contribution is superimposed to the transverse plasmon. We rather envision the following explanation for points (i) and (ii):

Polydispersity of our sample generates a distribution of aspect ratios. The longitudinal plasmon resonance is more sensitive to the aspect ratio than the transverse one. This translates into an inhomogenous broadening of the longitudinal plasmon that alters the peak intensity ratio of the experimental spectrum compared to the calculated one.

From a microscopic point of view, the peak width of both longitudinal and transverse mode is influenced also by surface damping effects when the nanoparticle size becomes comparable to the mean free path of Gold $(50 \mathrm{~nm})$ [26] therefore, in addition to polydispersity, surface dumping effects as well contribute to the observed spectral width both in the longitudinal and in the transverse peak. However, this effect is accounted for in the calculated spectra since a size corrected and anisotropic dielectric constant is used, as mentioned in the experimental section. Therefore, we would infer that point (ii) is due to the polydispersity of our sample, as hypothesized above.

\section{Near Field DDA Simulations of Bare Nanorods}

DDA calculations on NRs with the geometrical parameters defined in Fig. 2 were carried out and are described in the following. The square of the electric field around the rod is plotted in Fig. 3. As shown, the longitudinal mode (excited at $785 \mathrm{~nm}$ ) is characterized by an electric field intensity that reaches values about 10 times higher than the transverse one (excited at $514 \mathrm{~nm}$ ). Moreover, the longitudinal mode is mainly localized at the caps of the rod, whereas the transverse one spreads on the particle sides. Numerically, the SERS enhancement averaged over the whole particle surface, turned out to be about $10^{4}$ and
Fig. 3 Calculated distribution of the square of the electromagnetic field around a single particle at (a) $514 \mathrm{~nm}$ for the transverse plasmon and at (b) $785 \mathrm{~nm}$ for the longitudinal plasmon
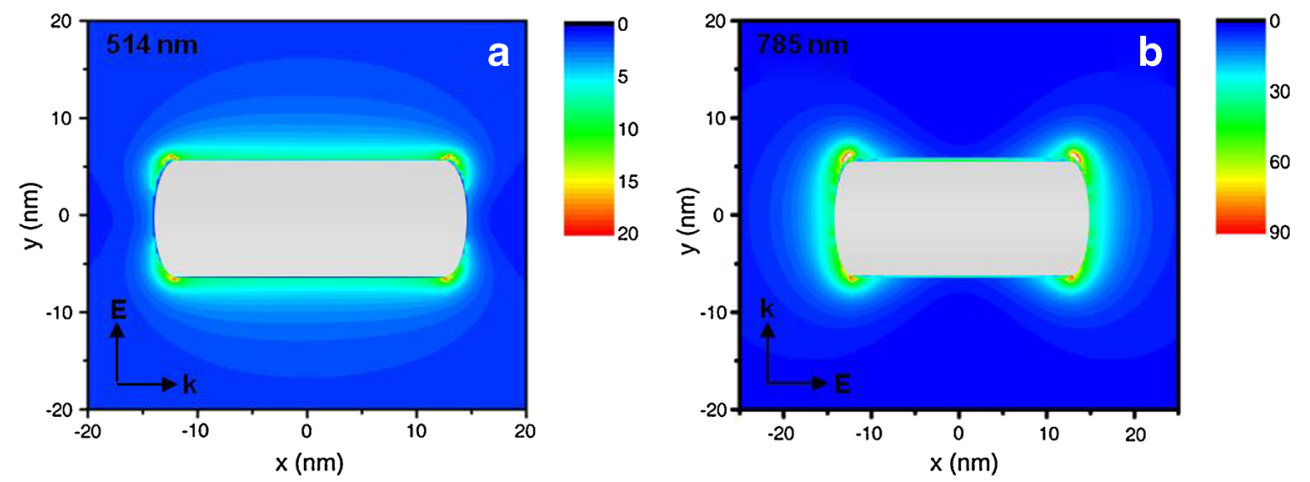
$10^{2}$, respectively, therefore corresponding to a longitudinal/transverse ratio of about 100 .

Optical and Morphological Characterization of Functionalized Au NRs

\section{IR and TEM}

Infrared spectroscopy of thiol functionalized gold nanostructures is routinely used in order to establish the binding of thiols and to study the structure of the organic ligand shell [27]. Figure 4 shows FT-IR spectra of PEP+C3SH (black line), PEP+C3SH functionalized Au NRs (PEP+C3-NRs, red line), PEP+C12SH functionalized Au NRs $(\mathbf{P E P}+\mathbf{C 1 2}-$ NRs, green line), and CTAB stabilized Au NRs (blue line). In the IR spectrum of CTAB stabilized NRs (blue line), the vibrational modes characteristic of CTAB are clearly visible. The methylene C-H stretching modes appear at 2,918 and $2,850 \mathrm{~cm}^{-1}$, whereas the alkyl bending vibrations appear at $1,480 \mathrm{~cm}^{-1}$. When NRs are functionalized with PEP+C3SH (red line) or PEP+C12SH (green line), in the IR spectrum new vibrational modes emerge that we associate to the PEP+ moiety (black line): the $\mathrm{C}=\mathrm{C}$ aromatic stretching at $1,604 \mathrm{~cm}^{-1}$ and the $\mathrm{C}-\mathrm{N}$ stretching at $1,182 \mathrm{~cm}^{-1}$. The frequencies of the signals originating from $\mathrm{C}-\mathrm{H}$ stretching modes have been often used to establish the degree of order and the extent to which the all-trans conformation is adopted by aliphatic chains in self-assembled monolayers on Au flat surface and nanoparticles [27, 28]. For example, NP coated with alkanethiols containing six or more methylene groups in the chain present the methylene $\mathrm{C}-\mathrm{H}$ symmetric stretching mode at $2,850 \mathrm{~cm}^{-1}$, characteristic of an all-trans conformation of the alkyl chains. Instead, shorter alkanethiols coating usually present several gauche defects, observable through a shift of this mode signal to higher frequency.

The methylene $\mathrm{C}-\mathrm{H}$ symmetric stretching mode is at 2,850 $\mathrm{cm}^{-1}$ for both PEP+C3-NRs and PEP+C12-NRs,

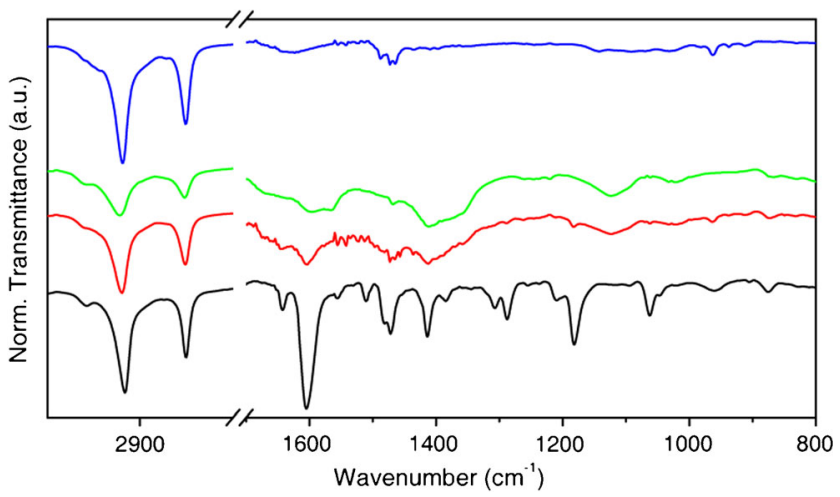

Fig. 4 FT-IR spectra of NRs functionalized with PEP+ moiety: PEP+ C3SH (black), PEP+C3-NRs (red), PEP+C12-NRs (green), and CTAB stabilized NRs (blue) consistent with an all-trans conformation of the alkyl chains in these systems. This finding suggests that, in both cases, the alkyl linkers between the thiol group and the PEP+ moieties are fully extended. Therefore, the distance between the PEP+ moiety and the surface-anchoring thiol group is ca. $1.6 \mathrm{~nm}$ for $\mathbf{P E P}+\mathbf{C 1 2 S H}$ and ca. $0.5 \mathrm{~nm}$ for PEP+C3SH.

\section{$U V-V I S$}

In Fig. 5a and b, the UV-visible extinction spectra of Au NRs before and after the functionalization procedure with PEP+ C3SH and PEP+C12SH are shown. Both water soluble $\mathbf{P E P}+\mathbf{C 3 S H}$ and PEP+C12SH (red traces in Fig. 5a and b) exhibit a narrow absorption band with a maximum at 431 and at $428 \mathrm{~nm}$, respectively.

After the functionalization process, both longitudinal and transverse plasmon absorption features are retained for NRs despite repeated washing by centrifugation/re-dispersion cycles (up to 10 times) to remove free dye molecules in solution. Interestingly, performing the same procedure on CTABstabilized Au NRs causes the nanoparticles to aggregate as suggested by the flattening and broadening of the longitudinal plasmon band in the UV-visible spectrum (data not shown). This means that the PEP+ functionalization somehow prevents NRs aggregation. A slight broadening of longitudinal and transverse plasmon absorption bands is clearly visible for $\mathrm{Au}$ NRs functionalized with PEP+C3SH (PEP+C3-NRs) and PEP+C12SH (PEP+C12-NRs). In addition, a 7-nm blue shift and a $30-\mathrm{nm}$ red shift in the longitudinal peak maximum can be observed in the green traces of Fig. 5a and $\mathrm{b}$, respectively. The interaction of Au NRs with thiol groups typically results in a dampening and a broadening of the plasmon absorption bands, as described in the literature [11, 29]. Furthermore, it should be emphasized that replacing CTAB with either PEP+C3SH or PEP+C12SH, i.e., with heterocyclic dyes possessing large nonlinear responses, implies a strong increase in the polarizability of the capping layer and contributes to the shift of the plasmon resonances.

PEP+C3-NR and PEP+C12-NR extinction spectrum shows in both cases a weak shoulder around $400 \mathrm{~nm}$, ascribable to the dye, slightly blue shifted with respect to the absorption band of the free PEP+ moiety. Despite the difficulty in unequivocally defining the band position of the dye after binding to Au NRs, due to the low absorbance value, the apparent blue shift of the band suggests that the adsorbed dye molecules interact with each other like they do in H-type aggregates of PEP + commonly reported in the literature [20, 21]. Another possible origin of the changes in the molecular resonance may arise from some degree of hybridization between molecular and plasmon excitations in cases in which the two are close to coincidence. Such phenomenon has been recently analyzed theoretically [30]. 
Fig. 5 UV-visible absorption spectra of bare NRs in water (black trace), dye (red trace), and dye functionalized NRs (green trace) for the case of PEP+C3SH (a) and for PEP+C12SH (b)
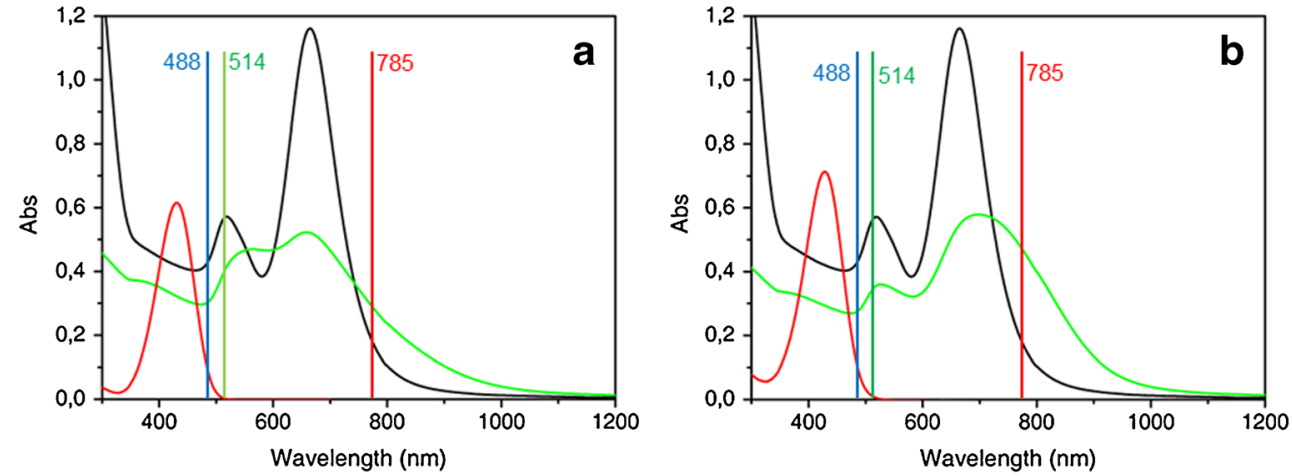

The TEM images of the Au NRs in water before functionalization (Fig. 6a) and after the anchoring of PEP+ C3SH (Fig. 6b) and PEP+C12SH (Fig. 6c) further confirm that no morphological changes occur after binding. The PEPfunctionalized $\mathrm{Au}$ NRs are well separated and exhibit minimal changes in shape and size.

\section{Surface Enhanced Raman Spectra of PEP $+C 3 S H$ and PEP + C12SH Bound to NRs and Deposited on a Glass Slide}

Here we aim to the identification and assignment of the Raman bands of PEP+ in powders and attached to NRs: we worked on solid samples since the Raman signal is much stronger than in the measurements in solution described in the next section.

Due to the PEP+ fluorescence, spectra of dye powders could be collected only at $785 \mathrm{~nm}$. Spectra at several wavelengths $(785,514$, and $488 \mathrm{~nm})$ were collected from NRs functionalized with PEP+C3SH and PEP+C12SH deposited on a glass slide by drop casting. As shown in Fig. 5, the 488$\mathrm{nm}$ and the 514-nm laser excitation lines are close to resonance with the absorption band of the molecules. Therefore, we can refer to these spectra as surface enhanced resonance Raman spectra (SERRS). When the laser excitation is not in electronic resonance with the molecular system, as for the 785-nm laser excitation line, we can refer to the observed enhanced spectra as surface enhanced Raman spectra (SERS).
Peak frequencies and assignment associated to the pyridinium and the pyrrole groups are given in Table 1 following the literature [31-34]. Figure 7 shows typical spectra (averaged over 5 points) of the powders of the $\mathbf{P E P}+$ dyes and of the functionalized NRs deposited on a glass slide, normalized with respect to cyclohexane. If we reasonably assume that the number of molecules probed in the dye powders is higher than in PEP+C3SH-NR and PEP+C12SH-NR ones, the spectra evidence a remarkable SERS effect at $785 \mathrm{~nm}$.

\section{Surface Enhanced Raman and Resonance Raman Spectra of PEP + C3SH and PEP + C12SH Bound to NRs in Solution}

SERS/SERRS experiments were repeated on functionalized NRs in aqueous solutions to study the properties of an ensemble of non-interacting nanoparticles. In order to ascertain whether significant NR aggregation occurs in solution, we performed dynamic light scattering (DLS) measurements. The particle size, measured by DLS, was $33 \pm 5 \mathrm{~nm}$ for bare NRs, close to the long axis length of the NRs measured by TEM. For functionalized NRs the rather poor quality of the DLS measurements does not allow us to rule out the possible presence of limited amounts of dimers. However, the results suggest that extensive aggregation did not take place. Therefore, our SERRS/SERS spectra should not be significantly affected by aggregation or plasmon coupling effects and comparison with DDA simulations of isolated NR is meaningful.
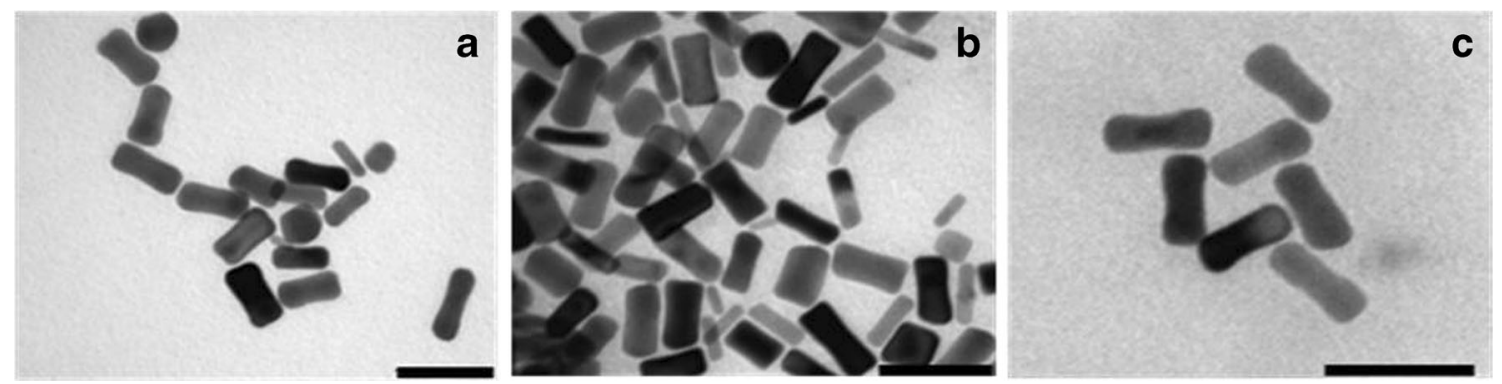

Fig. 6 TEM images of (a) Au NRs, (b) PEP+C3-NRs, and (c) PEP+C12-NRs purified by dye excess at $12 \mathrm{~h}$ (scale bar $50 \mathrm{~nm}$ ) 


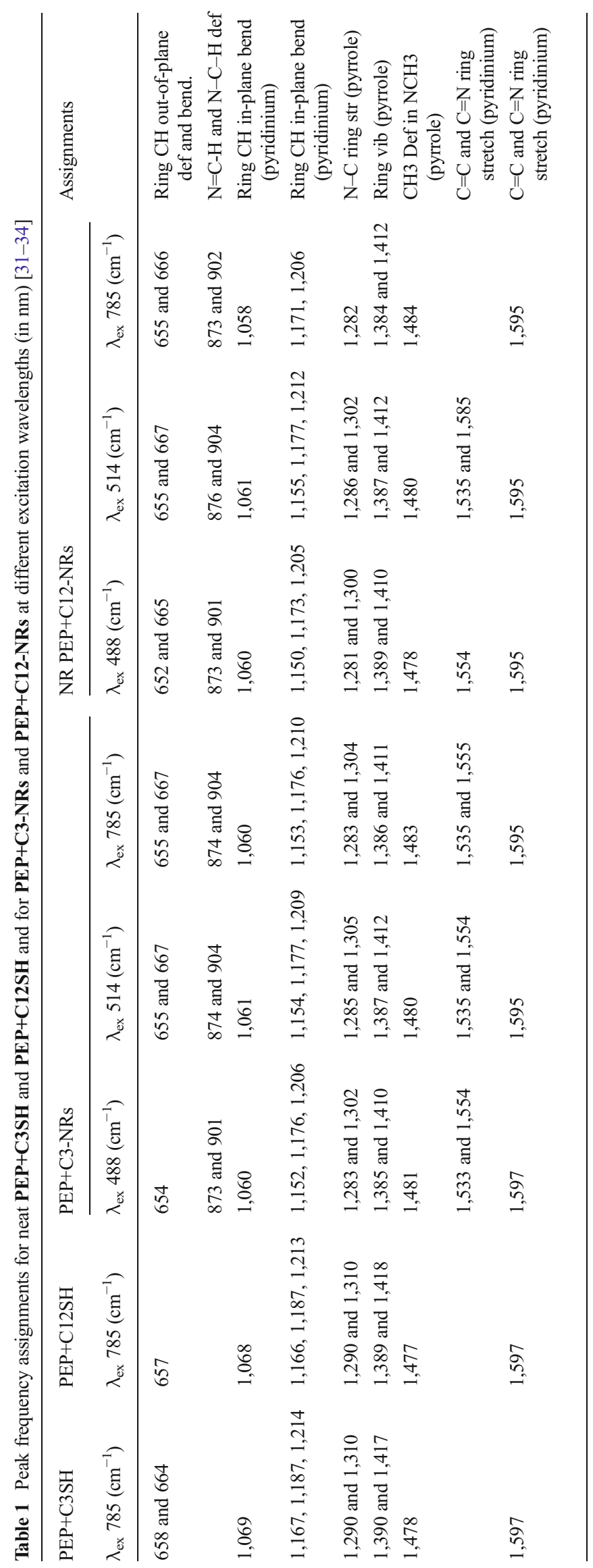


Fig. 7 a SERRS and SERS spectra of PEP+C3SH powders (black) and bound to NRs (deposited on a glass slide) at different laser excitation. b SERRS and SERS spectra of $\mathbf{P E P}+\mathbf{C 1 2 S H}$ powders (black) and bound to NRs (deposited on a glass slide) at different laser excitation
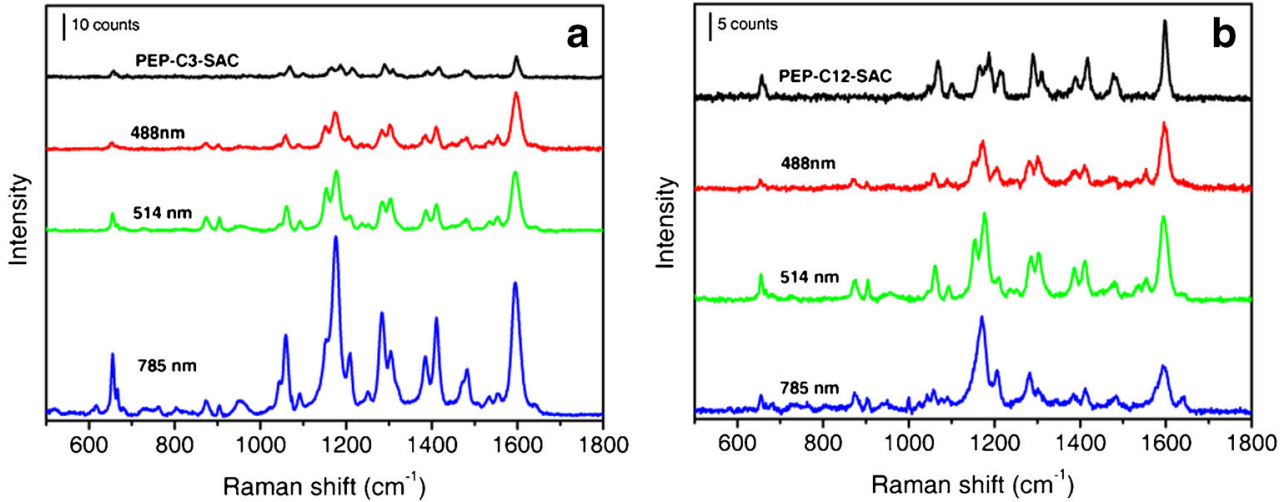

SERS enhancement factors (EF) can be evaluated by comparing the intensity of a selected Raman band of PEP+C3SH bound to NR with the intensity of the same band when the dye is free in solution, upon normalization by the number of illuminated molecules. Although, thanks to fluorescence quenching, SERS spectra of PEP+C3SH-NR are measurable at all excitation wavelengths used (see spectra in Fig. 8), fluorescence hindered the acquisition of PEP+ C3SH spectra in solution at 514 and $488 \mathrm{~nm}$. Therefore the procedure above could be used to determine the EF only at $785 \mathrm{~nm}$ excitation: details of the method are provided in the following. Similar considerations apply to PEP+C12SH-NR.

EFs are obtained by using the expression [15]:

$E F=\frac{I_{S E R S}}{I_{\text {Raman }}} \times \frac{M_{P E P+}}{M_{N R-P E P+}}$

where $M_{P E P+}$ is the concentration of $\mathbf{P E P}+\mathbf{C} 3 S H$ molecules sampled in the reference solution, $M_{N R-P E P+}$ is the

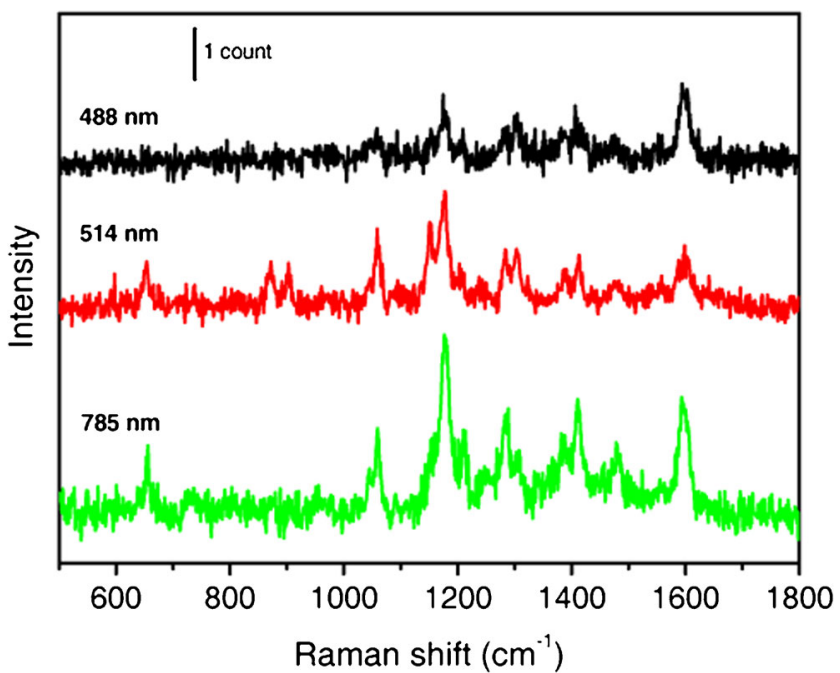

Fig. 8 SERRS and SERS spectra, in solution, of PEP+C3-NRs at different laser excitation concentration of PEP+C3SH or PEP+C12SH molecules bound to NRs in water solution, $I_{S E R S}$ is the intensity of a vibrational mode in the surface-enhanced spectrum, and $I$ Raman is the intensity of the same mode in the Raman spectrum. Vibrational modes used to determine enhancement factors include the ring $\mathrm{CH}$ out-of-plane deformation and bending $\left(655 \mathrm{~cm}^{-1}\right)$ and the in-plane bending $\left(1,069 \mathrm{~cm}^{-1}\right.$ and $\left.1,180 \mathrm{~cm}^{-1}\right)$ modes, the $\mathrm{N}-\mathrm{C}$ ring stretching mode $\left(1,289 \mathrm{~cm}^{-1}\right)$, the ring vibration mode $\left(1,415 \mathrm{~cm}^{-1}\right)$.

$M_{N R-P E P+}$ has been evaluated by determining the concentration of NRs in solution and the number of PEP+ molecules adsorbed per NR. To evaluate the concentration of NRs in solution, we considered the extinction coefficients calculated by Orendorff et al. [35] and related to the corresponding NRs aspect ratio and longitudinal plasmon peak maximum wavelength. The extinction coefficient $\left(\varepsilon_{N R}\right)$ at the peak of the longitudinal plasmon band increases linearly with increasing the wavelength $\left(\lambda_{\text {long }}\right)$ of the longitudinal plasmon band [35]. From interpolation of the reported data, the following empirical relation can be derived:

$\varepsilon_{N R}\left(\times 10^{9} M^{-1} \mathrm{~cm}^{-1}\right)=-5+0.012 \lambda_{\text {long }}(\mathrm{nm})$

Since our NRs present a longitudinal plasmon band at $\lambda_{\text {long }}=670 \mathrm{~nm}$, the calculated extinction coefficient $\varepsilon_{N R}$ is $3 \times 10^{9} \mathrm{M}^{-1} \mathrm{~cm}^{-1}$. Using the Lambert-Beer law, we calculated NRs concentration $\left(C_{N R}\right)$ in water as $2 \times 10^{12}$ particles $/ \mathrm{mL}$. The Surface area of a single NR is calculated as $S A_{N R}=2 \pi\left(r^{2}+\right.$ $\left.h^{2}\right)+2 \pi r l=1,193 \mathrm{~nm}^{2}=1.09 \times 10^{-15} \mathrm{~m}^{2}$, where $r=6 \mathrm{~nm}$ is the radius, $l=25 \mathrm{~nm}$ and $h=2 \mathrm{~nm}$ (see inset in Fig. 2a). To calculate the number of molecules bound to a single NR, we considered the area occupied by a single molecule [36]. The footprint of a PEP+ molecule lying edge-on is on the order of $0.5 \mathrm{~nm}^{2}$ [21]. If we consider a uniform binding of the thiol on all the surface, the number of molecules for a single NR is given by the ratio of the surface area of a NR to the footprint of the thiol $S A_{N R} / S A_{\text {thiol }}$, which is about 2400 . Finally, $M_{N R-P E P+}$ 
turns out to be about $7 \cdot 10^{-6}$ and $\mathrm{EF}(785)$ is in the order of $10^{4}$ for both PEP+C3SH-NR and PEP+C12SH-NR.

Concerning the other two excitation wavelengths (488 and $514 \mathrm{~nm}$ ), we normalized the data, along with those at $785 \mathrm{~nm}$, with respect to cyclohexane bands lying within $30 \mathrm{~cm}^{-1}$ of the PEP+C3-NR bands: by this method, the $\omega^{4}$ dependence is accounted for as well as instrument sensitivity issues. Clearly, on comparing normalized intensities at different excitation wavelengths, the molecular resonance effects do not cancel out.

A parameter $R$, which corresponds to the ratio $\frac{E F(785)}{E F(488,514)}$ except for molecular resonance effects, can be defined:

$R(488,514)=\frac{I^{\mathrm{PEP}+\mathrm{C} 3 \mathrm{SH}-\mathrm{NR}}(785) \quad I^{\text {Cyclohexane }}(488,514)}{I^{\text {Cyclohexane }}(785) \quad I^{\mathrm{PEP}+\mathrm{C} 3 \mathrm{SH}-\mathrm{NR}}(488,514)}$

The results at 488,514 , and $785 \mathrm{~nm}$ are summarized in Table 2.

The values of $R$ in Table 2 indicate that the normalized intensities at $785 \mathrm{~nm}$ are about 3-7 times higher than the normalized intensities at 514 or $488 \mathrm{~nm}$ excitation.

We attempted to perform SERS measurements with a nonresonant probe, that is 4-mercapto-pyridine (4-MPy), adsorbed on NRs by exciting at 514 and at $758 \mathrm{~nm}$. 4-MPy absorbs in the UV (324 $\mathrm{nm})$, hence molecular resonance enhancement of Raman spectra does not occur at 514 nor at $785 \mathrm{~nm}$.

A $0.022-\mathrm{M}$ aqueous solution of 4-MPy was used as a reference for evaluation of the SERS enhancement. Notice that the final analyte concentration in a $2 \times 10^{12}$ particle $/ \mathrm{mL}$ NRs solution was $10^{-6} \mathrm{M}$, i.e., almost four orders of magnitude less concentrated than the reference 4-MPy solution. Aqueous SERS solutions were equilibrated under ambient conditions for $10 \mathrm{~min}$ prior to spectral analysis. At 514-nm excitation, no appreciable SERS signal is observed for 4-MPy bound to NRs (data not shown) due to a small EF, insufficient to compensate for the nano-molar concentration in the NR

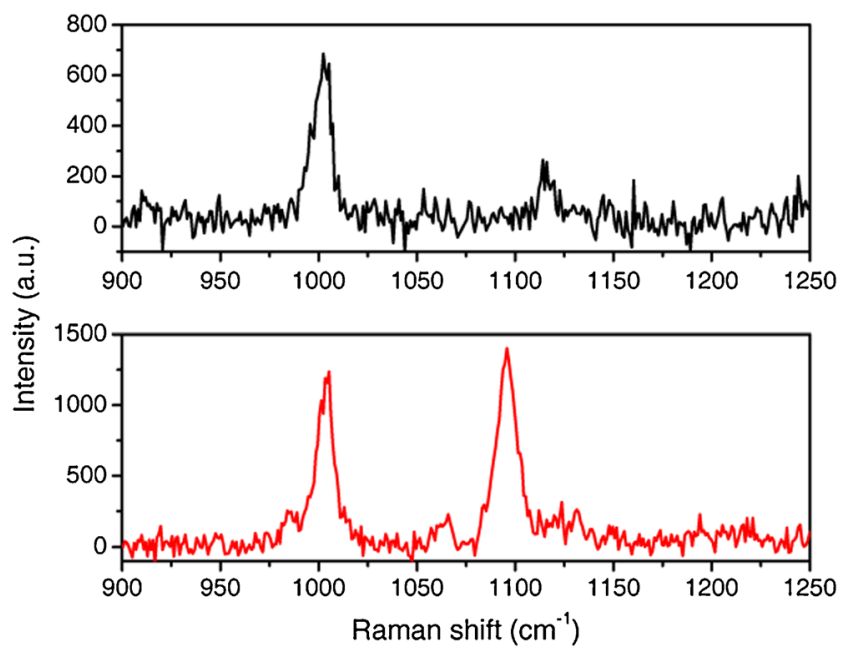

Fig. 9 SERS spectra excited at $785 \mathrm{~nm}$, of a $0.022 \mathrm{M}$ aqueous solution of 4-MPy (black trace) and $10^{-6} \mathrm{M}$ aqueous solution of 4-MPy bound to NRs (red trace)

solution. Conversely, Raman signals of 4-MPy bound to NRs are strongly amplified when excited by 785 -nm laser wavelength (Fig. 9, lower panel).

Characteristic ring breathing modes are observable at $1,004 \mathrm{~cm}^{-1}(v(\mathrm{C}-\mathrm{C})$ mode $)$ and at $1,119 \mathrm{~cm}^{-1}(v(\mathrm{C}-\mathrm{S})$ mode $)$ in the spectrum of the reference solution (Fig. 9, upper panel). The latter mode is remarkably shifted in 4-MPy bound to NRs $\left(1,094 \mathrm{~cm}^{-1}\right)$ and experiences a dramatic increase in intensity compared with that in the Raman spectrum of the solution. Similar enhancement has been reported for 4-Mpy adsorbed on other metal substrates, which was interpreted by coordination of 4-Mpy with the metal surface through the sulfur atom [37].

Notice that, despite the much lower concentration (by almost four orders of magnitude), the SERS spectrum exhibits a better signal-to-noise ratio than the solution one. The evaluated $\mathrm{EF}$ for the $v(\mathrm{C}-\mathrm{C})$ ring breathing mode at $1,004 \mathrm{~cm}^{-1}$ and the $v(C-S)$ ring breathing mode at $1,119 \mathrm{~cm}^{-1}$ are $3.7 \times$ $10^{4}$ and $1.1 \times 10^{5}$, respectively, in reasonable agreement with the PEP+C3-NRs and PEP+C12-NRs measurements. The very fact that an appreciable SERS signal could not be
Table $2 \mathrm{EF}$ and $R$ factors of $\mathbf{P E P}+\mathbf{C} 3 \mathrm{SH}$ and $\mathbf{P E P}+\mathbf{C 1 2 S H}$ on $\mathrm{Au}$ NRs for different vibrational modes

\begin{tabular}{|c|c|c|c|c|c|}
\hline \multirow[t]{2}{*}{ Band $\left(\mathrm{cm}^{-1}\right)$} & \multicolumn{2}{|c|}{$\mathrm{PEP}+\mathrm{C} 3-\mathrm{NR}$} & \multicolumn{2}{|c|}{$\mathrm{PEP}+\mathrm{C} 12-\mathrm{NR}$} & \multirow{2}{*}{$\begin{array}{l}\text { 4-Mpy-NR } \\
\text { EF (785) }\end{array}$} \\
\hline & R (488) & $\mathrm{R}(514)$ & $\mathrm{EF}(785)$ & & \\
\hline 655 & & & $1.6 \times 10^{4}$ & $1.4 \times 10^{4}$ & \\
\hline 1,069 & 3.7 & 3.2 & $1.1 \times 10^{4}$ & $1.9 \times 10^{4}$ & \\
\hline 1,180 & 3.0 & 3.9 & $1.7 \times 10^{4}$ & $9.3 \times 10^{3}$ & \\
\hline 1,289 & 2.7 & 4.3 & $1.0 \times 10^{4}$ & & \\
\hline 1,415 & 4.8 & 7.2 & $9.0 \times 10^{3}$ & & \\
\hline 1,004 & & & & & $3.7 \times 10^{4}$ \\
\hline 1,119 & & & & & $1.1 \times 10^{5}$ \\
\hline
\end{tabular}


detected when exciting at $514 \mathrm{~nm}$ confirms that the EF at resonance with the transverse plasmon is at least one or two orders of magnitude weaker than at resonance with the longitudinal one. Since the normalized intensities defined by $\mathrm{R}(488,514)$ in Table 2 are in the range $3-7$, the results reported above for $\mathbf{P E P}+\mathbf{C 3}$-NRs can only be explained considering that resonance with the molecular excitation contributes an enhancement of the Raman signal comparable to that due to resonance with the transverse plasmon.

Finally, Table 2 shows that EFs of PEP+C3-NRs and PEP+C12-NRs do not exhibit any clear distance dependence although it would be expected in the range of a factor of 2-4 from literature data [38]. We tentatively explain this apparent lack of distance dependence with the following two arguments:

- the packing density of C3PEP+ and C12PEP+ is assumed to be equal, but it could be limited in PEP+C3NRs by the bulky heterocyclic heads. In PEP+C12-NRs the longer alkyl chains may help achieving a more compact packing.

- the SERS measurements are taken from different samples (the same batch of NRs, a part functionalized with PEP+ C3-NRs and a part with PEP+C12-NRs) and therefore reproducibility issues throughout the functionalization/ purification processes could contribute to hinder the expected distance dependence.

\section{Experimental}

\section{Materials}

Sodium borohydride $\left(\mathrm{NaBH}_{4}, \sim 99 \%\right)$, L-ascorbic acid (99\%), hydrogen tetrachloroaurate(III) trihydrate $\left(\mathrm{HAuCl}_{4}\right.$. $3 \mathrm{H}_{2} \mathrm{O}, \geq 99.9 \%$ ), cetyltrimethylammonium bromide (CTAB), and silver nitrate $\left(\mathrm{AgNO}_{3}, 99.9999 \%\right)$ were purchased from Aldrich. Cyclohexane ( $\geq 99.5 \%)$ was obtained from Fluka. Acetone (99.8 \%) was purchased from Carlo Erba. Stock ion solutions were prepared using deionized water (Millipore milli-Q Gradient A-10 system). $\mathrm{NH}_{4} \mathrm{OH}$ (30 \%) was purchased from Fluka. Absolute ethanol was purchased from Baker. Dry acetone was stored over $\mathrm{CaCl}_{2}$.

\section{Preparation of PEP $\pm C 3 S A C$ and $P E P \pm C 12 S A C$}

The two $\omega$-acetylthioalkyl derivatives of PEP+ were prepared according to Fig. 10 by microwave-promoted alkylation of 1-(pyrid-4-yl)-2-( $N$-methylpyrrol-2-yl)ethylene $(\mathbf{1})^{21}$ with the proper $\omega$-acetylthioalkyl bromide $\mathbf{2 a}$ [39] or $\mathbf{2 b}$ [40].

PEP+C3SAc. A solution of 1-(pyrid-4-yl)-2-(Nmethylpyrrol-2-yl)ethylene (1) (0.102 g, $0.55 \mathrm{mmol})$ and $\omega$ -

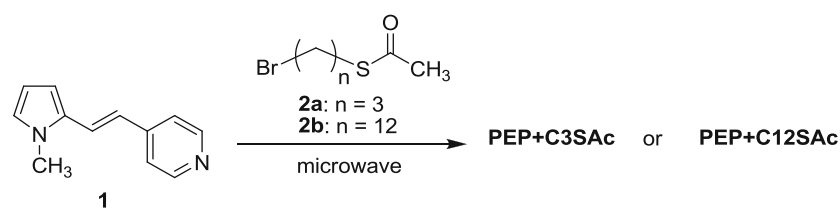

Fig. 10 Reaction scheme for the synthesis of $\mathrm{PEP}+\mathrm{C} 3 \mathrm{SAC}$ and $\mathrm{PEP}+$ C12SAC

acetylthiopropyl bromide $(0.218 \mathrm{~g}, 1.10 \mathrm{mmol})$ in dry acetonitrile $(3 \mathrm{~mL})$ was irradiated at $100 \mathrm{~W}$ for $2 \mathrm{~h}$ in a microwave reactor. The formed precipitate was collected by filtration to afford the product as a red solid $(0.131 \mathrm{~g}, 0.34 \mathrm{mmol}, 62 \%)$; ${ }^{1} \mathrm{H}-\mathrm{NMR}$ (DMSO- $d_{6}$ ) $\delta 8.75$ (d, J 7.0, 2H, pyridine ring), 8.14 (d, $J$ 7.0, 2H, pyridine ring), 7.94 (d, $J 15.9,1 \mathrm{H}$, vinylene), 7.12 (bs, 1H, pyrrole), 7.08 (d, $J 15.9,1 \mathrm{H}$, vinylene), 6.89 (dd, $J 3.5,2.2,1 \mathrm{H}$, pyrrole), 6.24 (dd, $J 4.1,3.2,1 \mathrm{H}$, pyrrole), 4.53 $\left(\mathrm{t}, J 7.7,2 \mathrm{H}, \mathrm{CH}_{2} \mathrm{~N}^{+}\right)$, and $3.80\left(\mathrm{~s}, 3 \mathrm{H}, \mathrm{NCH}_{3}\right)$; other signals are covered by solvent (2.5 ppm) and water (3.4 ppm) peaks; Anal. Calcd. for $\mathrm{C}_{17} \mathrm{H}_{21} \mathrm{BrN}_{2} \mathrm{OS}$ : C, 53.54; H, 5.55; N, 7.35; Found: C, 53.04; H, 5.68; N, 7.22.

PEP+C12SAc. A solution of 1-(pyrid-4-yl)-2-(Nmethylpyrrol-2-yl)ethylene (1) (0.184 g, $1.00 \mathrm{mmol})$ and $\omega$-acetylthiododecyl bromide $(0.890 \mathrm{~g}, 2.75 \mathrm{mmol})$ in dry acetone $(5 \mathrm{~mL})$ was irradiated at $110 \mathrm{~W}$ for $3 \mathrm{~h}$ in a microwave reactor. After cooling to room temperature the precipitate was filtered and washed with cold acetone, yielding the product as an orange solid (0.300 g, $0.590 \mathrm{mmol}, 59 \%)$; ${ }^{1} \mathrm{H}-\mathrm{NMR}$ (DMSO- $\left.d_{6}\right) \delta$ 8.80 (d, $J 6.5,2 \mathrm{H}$, pyridine ring), 8.18 (d, $J 6.5,2 \mathrm{H}$, pyridine ring), 7.90 (d, $J 15.8,1 \mathrm{H}$, vinylene), 7.12 (bs, $1 \mathrm{H}$, pyrrole), 7.08 (d, $J 15.8,1 \mathrm{H}$, vinylene), 6.90 (d, $J$ $3.7,1 \mathrm{H}$, pyrrole), 6.20 (d, $J 4.0,1 \mathrm{H}$, pyrrole), 4.45 (t, $J$ 7.2, $\left.2 \mathrm{H}, \mathrm{CH}_{2} \mathrm{~N}^{+}\right), 3.80\left(\mathrm{~s}, 3 \mathrm{H}, \mathrm{NCH}_{3}\right), 2.84(\mathrm{t}, J$ 7.0, $\left.2 \mathrm{H}, \mathrm{CH}_{2} \mathrm{~S}\right), 2.30(\mathrm{~s}, 3 \mathrm{H}, \mathrm{SAc}), 2.0-1.7$ (m, 2H, alkyl chain), 1.6-1.4 (m, 2H, alkyl chain), 1.4-1.0 (m, 16H, alkyl chain); Anal. Calcd. for $\mathrm{C}_{26} \mathrm{H}_{39} \mathrm{BrN}_{2} \mathrm{OS} \cdot 1 / 2 \mathrm{H}_{2} \mathrm{O}$ : C, 60.45; H, 7.80; N, 5.42; Found: C, 60.62; H, 7.85; $\mathrm{N}, 5.47$.

\section{Preparation of Water Soluble Au NRs}

The water soluble surfactant-capped $\mathrm{Au}$ NRs with longitudinal plasmon band at $670 \mathrm{~nm}$ were prepared by suitable modifications of previously reported seed-mediated growth approach [41, 42]. Such an approach involves two steps. The former concerns the preparation of a seed solution and was performed by mixing at room temperature two freshly prepared solutions of CTAB $(0.2 \mathrm{M})$ and $\mathrm{HAuCl}_{4}$. $3 \mathrm{H}_{2} \mathrm{O}\left(5 \times 10^{-4} \mathrm{M}\right)$. Then $0.6 \mathrm{~mL}$ of a $0.01-\mathrm{M}$ ice-cold aqueous solution of $\mathrm{NaBH}_{4}$ were added into the aforementioned mixture under vigorous stirring. Within a few seconds the colour of the solution turned from yellow-orange, ascribable to $\mathrm{CTAB}-\mathrm{Au}^{3+}$ complexes [43], to brown, 
indicating the formation of $\mathrm{Au}$ seeds (i.e., Au NPs smaller than $3 \mathrm{~nm}$ ). The seed solution was left stirring for $2 \mathrm{~h}$ and used within $5 \mathrm{~min}$ after stopping the stirring. The latter step involves the growth of such seeds to form Au NRs at room temperature. Three-milliliter growth solution was prepared by mixing CTAB $(0.24 \mathrm{mmol}), 65 \mu \mathrm{L}$ of acetone and $45 \mu \mathrm{L}$ of cyclohexane (able to influence the micelle morphology), $\mathrm{AgNO}_{3}(0.31 \mu \mathrm{mol})$, and further $\mathrm{Au}$ precursor $\left(\mathrm{HAuCl}_{4} \cdot 3 \mathrm{H}_{2} \mathrm{O}, 0.006 \mathrm{mmol}\right)$. Under gentle mixing, an ascorbic acid solution was added dropwise (ascorbic acid/Au ratio $=2$ ) to reduce $\mathrm{Au}(\mathrm{III})$ to $\mathrm{Au}(\mathrm{I})$ as indicated by the colour of the growth solution which turned from dark yellow $(\mathrm{Au}(\mathrm{III}))$ to colourless $(\mathrm{Au}(\mathrm{I}))$. At this point, the NR growth was promoted by adding $60 \mu \mathrm{L}$ of the seed solution and revealed by the appearance of a blueviolet color in a few minutes. In order to prevent the aggregation of $\mathrm{Au}$ NRs owing to standard purification by centrifugation procedure, the dye anchoring experiments were performed on the as prepared NR solution without further purification.

\section{Anchoring of PEP+ Dyes on Au NRs}

The dyes PEP+C3SAc and PEP+C12SAc are well soluble in ethanol and less soluble in water, especially for the derivative with the longer alkyl chain. The acetyl group prevents the oxidation of the terminal thiol group and it should be removed before the functionalization of Au NRs. In fact, it is well known from the literature that thioacetates react slowly with Au surfaces and organize in a less ordered SAM [44]. In a typical deprotection procedure, $100 \mu \mathrm{L}$ of $\mathrm{NH}_{4} \mathrm{OH}(30 \%)$ was added in a solution of dye in ethanol $10^{-3} \mathrm{M}$. The as prepared mixture was left to stand for $20 \mathrm{~min}$ and then evaporated to dryness by rotavapor [45]. Such precipitate was dispersed in $1 \mathrm{~mL}$ of Au NR solution leaving the mixture to stand for $12 \mathrm{~h}$. The dye-NR complex solution was purified upon repeated cycles of dissolution in water and centrifugation (by centrifuge 5430; Eppendorf, at $8,100 \mathrm{rpm}$, at $25^{\circ} \mathrm{C}$, for $15 \mathrm{~min}$ ) to wash out unbound surfactant and dye residuals.

\section{Instrumentation and Calculations}

The linear absorption spectra of bare and functionalized $\mathrm{Au}$ NRs were recorded using a Varian Cary 5000 UV-Vis-NIR scanning spectrophotometer in the 300-1,200 nm. NMR spectra were recorded on a Bruker AMX-200 and AMX-500 instruments operating at 200 and $500 \mathrm{MHz}$, respectively. Coupling constants are given in $\mathrm{Hz}$.

TEM investigations have been performed by a Jeol JEM1011 microscope operating at $100 \mathrm{kV}$. The specimens have been prepared by depositing a few drops of aqueous NP dispersions onto a carbon-coated copper grid and by allowing the solvent to evaporate. A systematic statistical analysis of
NR size distributions was performed on the basis of lowmagnification TEM images with the help of Axio Vision software. At least $100 \mathrm{NPs}$ were counted for each sample.

Mid-infrared spectra have been acquired with a PerkinElmer Spectrum One Fourier transform infrared (FTIR) spectrometer equipped with a DTGS (deuterated tryglicine sulfate) detector. The spectral resolution used for all experiments was $4 \mathrm{~cm}^{-1}$. For attenuated total reflection (ATR) measurements, the internal reflection element (IRE) was a three-bounce, $4 \mathrm{~mm}$ diameter diamond microprism. Cast films were prepared directly onto the internal reflection element, by depositing the solution of interest $(3-5 \mu \mathrm{L})$ on the upper face of the diamond crystal and allowing the solvent to evaporate completely.

Raman scattering experiments were conducted with two different instruments. The first one was a home-built microRaman system, based on a Triax-320 ISA spectrograph, equipped with a holographic $1,800 \mathrm{~g} / \mathrm{mm}$ grating and a CCD detector (Spectrum One ISA Instruments). The excitation source was a Spectra Physics $\mathrm{Ar}^{+}$laser (Stabilite 2017-06S) operating at 488 and $514.5 \mathrm{~nm}$. A Kaiser Optical System holographic notch filter was used to reduce the stray-light level. An Olympus BX 40 optical microscope equipped with a $50 \times / 0.75$ objective was optically coupled to the spectrograph. To avoid optical damage to the sample, the power of the exciting radiation was maintained between 0.15 and $0.5 \mathrm{~mW}$. The Raman spectra were recorded with 10 acquisitions, each of $5 \mathrm{~s}$, between 400 and $1,800 \mathrm{~cm}^{-1}$ and with an instrumental resolution of about $2 \mathrm{~cm}^{-1}$. The second instrument was a Renishaw RM2000 microRaman spectrometer, coupled with a diode laser source emitting at $785 \mathrm{~nm}$. Sample irradiation was accomplished using the $50 \times$ microscope objective of a Leica Microscope DMLM. The beam power was about $0.28 \mathrm{~mW}$. Raman scattering was filtered by a double holographic Notch filter system and collected by an air cooled CCD detector. The Raman spectra were recorded with 3 acquisitions, each of $30 \mathrm{~s}$, between 400 and $1,800 \mathrm{~cm}^{-1}$. All spectra were calibrated with respect to a silicon wafer at $520 \mathrm{~cm}^{-1}$.

For SERS/SERRS measurements, PEP+ functionalized NRs were either measured in solutions or deposited by drop casting on glass substrates. In order to compare the signal measured at different wavelengths, Raman spectra were normalized by using cyclohexane as an intensity standard following the method of Ref. [46].

\section{DDA Calculations}

The local field was calculated by the Discrete Dipole Approximation (DDA) method using the software developed by Draine and Flatau (DDSCAT 7.1 and the relative DDFIELD code) $[47,48]$. The DDA is one of the most frequently used method to calculate the optical properties and the local field of 
metal nanostructures of arbitrary shape [49-51]. In DDA, one can account for the structure of interest, usually called "target", by a cubic array of $\mathrm{N}$ polarizable points (i.e. $\mathrm{N}$ cubic dipoles). In this work, the target consisted in a cylinder with two hemispheroidal caps, with a main axis of $29 \mathrm{~nm}$ and a minor axis of $12 \mathrm{~nm}$ (inset in Fig. 2a). We used 173112 dipoles for the target, corresponding to an interdipole spacing of $0.27 \mathrm{~nm}$. For metal particles in the 2-200-nm-size range, an error smaller than $10 \%$ is usually achieved using a number of dipoles at least of the order of $10^{4}$ and using interdipole spacing much smaller than the wavelengths of interest. The effect of solvent has been accounted for through the refractive index of the non-absorbing matrix, that is $n=1.334$ for water. The refractive index of water and the Au dielectric constants from Palik were used [52]: we adopted a size-corrected dielectric constant, as reported in ref. [53]. Such dependence is due to the conduction electrons mean free path being comparable to particles size along the direction of polarization promoted by the electromagnetic field. [50, 53, 54]. The size correction has been treated separately for transverse and longitudinal excitation, since the mean free path is different in the two cases. Hence the dielectric constant is anisotropic due to the different size correction applied.

Simulated extinction spectra include the orientational average. The average has been worked out over the three possible orientations corresponding at the three situations in which the three rod axes are, one by one, parallel to the (linearly polarized) incoming beam.

As for the near-field calculations, the incident wavelength was 514 or $785 \mathrm{~nm}$ for the electric field parallel to the transverse or longitudinal axis of the rod, respectively. We calculated the square of the electric field by the DDFIELD code and plotted it in the plane that bisects the rod. Near-field was calculated at $0.5 \mathrm{~nm}$ from the surface of the NRs.

\section{Conclusions}

In this study, we measured Raman spectra of the heteroaromatic positively charged PEP+ dyes bound to $\mathrm{Au}$ NRs through linear alkyl chains of different lengths ( 3 or 12 Carbon atoms) terminated with a thiol group. Measurements were realized using the 488, 514, and 785-nm laser excitation lines to study the effect of the laser resonance with the two plasmon modes of NRs and with the absorption band of the molecules.

We observed that the amplification of the Raman signals is higher when the laser excitation wavelength is resonant with the longitudinal plasmon mode. Raman spectra performed exciting at 514-nm are only 4-7 times lower in intensity compared with the 785-nm excitation, indicating that the resonance condition of the 514-nm laser excitation with the absorption band of the dye strongly contributed to the amplification of the Raman signal.

To strengthen the previous experimental evidence we used 4-mercaptopyridine (4-MPy) as a standard analyte. Since it absorbs in the UV region, resonance with molecular excitations does not take place at 514 and $785 \mathrm{~nm}$. EF measured at $785 \mathrm{~nm}$ turned out to be about $4 \cdot 10^{4}$ for the ring breathing mode at $1,004 \mathrm{~cm}^{-1}$. In addition, the fact that at $514 \mathrm{~nm}$ the signal was not detectable confirms that the $\mathrm{EF}$ at resonance with the transverse plasmon is at least one order of magnitude lower than the longitudinal one.

In the light of these considerations, the resonance with the absorption band of PEP+ moiety is the main contribution to the enhancement in the Raman signal of the dye observed at 514-nm laser excitation.

Finally, this work evaluated by SERS the effectiveness of gold NRs as plasmonic enhancers of the optical properties of $\mathrm{PEP}+$ dyes under molecular resonant and non-resonant conditions. Since both SERS and two-photon absorption depend on the forth power of the local electric field, and since PEP+ dyes are well-known to possess remarkable nonlinear optical properties, the results presented in this paper are also expected to open the way to the improvement of nonlinear optical responses.

Acknowledgments The authors thank Dr. M. Muniz-Miranda (Chemistry Department of the University of Firenze) for his assistance with the Renishaw RM2000 microRaman apparatus and Dr. R. Signorini (Department of Chemical Sciences of the University of Padova) for stimulating discussions and a critical reading of the manuscript. Financial support from the Italian Ministry for Education, University and Research (MIUR) through the PRIN 2009 project "Novel materials and processes for plasmon-based sensors", from the University of Padova through the PLATFORM project "PLAsmonic nano-Textured materials and architectures FOR enhanced Molecular Sensing" and from the Italian Interuniversity Consortium for Science and Technology of Materials (INSTM) through the PROMO Programme is gratefully acknowledged.

Open AccessThis article is distributed under the terms of the Creative Commons Attribution License which permits any use, distribution, and reproduction in any medium, provided the original author(s) and the source are credited.

\section{References}

1. Kneipp K, Kneipp H, Kneipp J (2006) Surface-enhanced Raman scattering in local optical fields of silver and gold NanoaggregatesFrom single-molecule raman spectroscopy to ultrasensitive probing in live cells. Acc Chem Res 39:443-450

2. Kneipp K, Kneipp H, Manoharan R, Itzkan I, Dasari RR, Feld MS (1998) Surface-enhanced Raman scattering (SERS)-a new tool for single molecule detection and identification. Bioimaging 6:104-110

3. Kneipp K (2007) Surface-enhanced Raman scattering. Phys Today 60(11):40-46

4. Diebold ED, Mack NH, Doorn SK, Mazur E (2009) Femtosecond laser-nanostructured substrates for surface-enhanced Raman scattering. Langmuir 25:1790-1794 
5. Yan B, Thubagere A, Ranjith Premasiri W, Ziegler LD, Dal Negro L, Reinhard BM (2009) Engineered SERS substrates with multiscale signal enhancement: nanoparticle cluster arrays. ACS Nano 3:11901202

6. Yang M, Alvarez-Puebla RA, Kim HS, Aldeanueva-Potel P, LizMarzan LM, Kotov NA (2010) SERS-active gold lace nanoshells with built-in hotspots. Nano Lett 10:4013-4019

7. Bechelany M, Brodard P, Elias J, Brioude A, Michler J, Philippe L (2010) Simple synthetic route for SERS-active gold nanoparticles substrate with controlled shape and organization. Langmuir 26: 14364-14371

8. Jeong DH, Zhang YX, Moskovits M (2004) Polarized surface enhanced Raman scattering from aligned silver nanowire rafts. J Phys Chem B 108:12724-12728

9. Jackson JB, Halas NJ (2004) Surface-enhanced Raman scattering on tunable plasmonic nanoparticle substrates. PNAS 101:17930-17935

10. Camden JP, Dieringer JA, Zhao J, Van Duyne RP (2008) Controlled plasmonic nanostructures for surface-enhanced spectroscopy and sensing. Acc Chem Res 41:1653-1661

11. Link S, El-Sayed MA (2000) Shape and size dependence of radiative, non-radiative and photothermal properties of gold nanocrystals. Int Rev Phys Chem 19:409-453

12. Nikoobakht B, Wang J, El-Sayed MA (2002) Surface-enhanced Raman scattering of molecules adsorbed on gold nanorods: offsurface plasmon resonance condition. Chem Phys Lett 366:17-23

13. Nikoobakht B, El-Sayed MA (2003) Surface-enhanced Raman scattering studies on aggregated gold nanorods. J Phys Chem A 107: 3372-3378

14. Orendorff CJ, Gole A, Sau TK, Murphy CJ (2005) Surface-enhanced Raman spectroscopy of self-assembled monolayers: sandwich architecture and nanoparticle shape dependence. Anal Chem 77:3261-3266

15. Orendorff CJ, Gearheart L, Janaz NR, Murphy CJ (2006) Aspect ratio dependence on surface enhanced Raman scattering using silver and gold nanorod substrates. Phys Chem Chem Phys 8:165-170

16. Aroca RF, Goulet PJG, dos Santos DS, Alvarez-Puebla RA, Oliveira ON (2005) Silver nanowire layer-by-layer films as substrates for surface-enhanced Raman scattering. Anal Chem $77: 378-382$

17. Guo H, Ruan F, Lu L, Hu J, Pan J, Yang Z, Ren B (2009) Correlating the shape, surface plasmon resonance, and surface-enhanced Raman scattering of gold nanorods. J Phys Chem C 113:10459-10464

18. Pal S, Depero LE, Alessandri I (2010) Using aggregates of gold nanorods in SER(R)S experiments: an empirical evaluation of some critical aspects. Nanotechnology 21:425701

19. Bradamante S, Facchetti A, Pagani GA (1997) Heterocycles as donor and acceptor units in push-pull conjugated molecules. J Phys Org Chem 10:514-524

20. Facchetti A, Abbotto A, Beverina L, Van Der Boom ME, Dutta P, Evmenenko G, Garks TJ, Pagani GA (2002) Azinium- $(\pi-$ bridge)-pyrrole NLO-phores: influence of heterocycle acceptors on chromophoric and self-assembled thin-film properties. Chem Mater 14:4996-5005

21. Facchetti A, Abbotto A, Beverina L, Van Der Boom ME, Dutta P, Evmenenko G, Pagani GA, Marks TJ (2003) Layer-by-layer selfassembled pyrrole-based donor-acceptor chromophores as electrooptic materials. Chem Mater 15:1064-1072

22. Abbotto A, Beverina L, Bradamante S, Facchetti A, Pagani GA, Bozio R, Ferrante C, Pedron D, Signorini R (2003) Design and synthesis of heterocyclic multi-branched dyes for two-photon absorption. Synth Met 136:795-797

23. Abbotto A, Beverina L, Bozio R, Bradamante S, Pagani GA, Signorini R (2001) Heterocycle-based materials for frequencyupconverted lasing. Synth Met 121:1755-1756

24. Abbotto A, Beverina L, Bozio R, Bradamante S, Ferrante C, Pagani GA, Signorini R (2000) Push-pull organic chromophores for frequency-upconverted lasing. Adv Mater 12:1963-1967
25. He GS, Yuan L, Prasad PN, Abbotto A, Facchetti A, Pagani GA (1997) Two-photon pumped frequency-upconversion lasing of a new blue-green dye material. Opt Commun 140:49-52

26. Link S, El Sayed MA (1999) Size and temperature dependence of the plasmon absorption of colloidal gold nanoparticles. J Phys Chem B 103:4212-4217

27. Hostetler MJ, Stokes JJ, Murray RW (1996) Infrared spectroscopy of three-dimensional self-assembled monolayers: $\mathrm{N}$-alkanethiolate monolayers on gold cluster compounds. Langmuir 12:3604-3612

28. Malicki M, Hales JM, Rumi M, Barlow S, McClary L, Marder SR, Perry JW (2010) Excited-state dynamics and dye-dye interactions in dye-coated gold nanoparticles with varying alkyl spacer lengths. Phys Chem Chem Phys 12:6267-6277

29. Jebb M, Sudeep PK, Pramod P, Thomas KG, Kamat PV (2007) Ruthenium(II) trisbipyridine functionalized gold nanorods. Morphological changes and excited-state interactions. J Phys Chem B 111:6839-6844

30. Ambjörnsson T, Mukhopadhyay G, Apell S, Käll M (2006) Resonant coupling between localized plasmons and anisotropic molecular coatings in ellipsoidal metal nanoparticles. Phys Rev B 73:085412

31. Obaid AY, Soliman MS (1990) Vibrational spectra and molecular force field of $\mathrm{N}$-methylpyridinium ion and some deuterated derivatives. Spectrochimica Acta Part A 46:1779-1791

32. Dubis AT, Grabowski SJ (2003) Infrared, density-functional theory, and atoms in molecules method studies on conformers of some 2-substituted 1H-pyrroles. J Phys Chem A 107:87238729

33. Gunasekaran S, Sankari G, Ponnusamy S (2005) Vibrational spectral investigation on xanthine and its derivatives - theophylline, caffeine and theobromine. Spectrochimica Acta Part A 61:117-127

34. Socrates G (2005) Infrared and Raman characteristic group frequencies. John Wiley \&Sons LTD

35. Orendorff CJ, Murphy CJ (2006) Quantitation of metal content in the silver-assisted growth of gold nanorods. J Phys Chem B 110:3990-3994

36. Bardhan R, Grady NK, Cole GR, Joshi A, Halas NJ (2009) Fluorescence enhancement by au nanostructures: nanoshells and nanorods. ACS Nano 3:744-752

37. Guo H, Ding L, Mo Y (2011) Adsorption of 4-mercaptopyridine onto laser-ablated gold, silver and copper oxide films: A comparative surface-enhanced Raman scattering investigation. J Mol Struct 991: 103-107

38. Compagnini G, Galati G, Pignataro S (1999) Distance dependence of surface enhanced Raman scattering probed by alkanethiol self-assembled monolayers. Phys Chem Chem Phys 1:2351-2353

39. Block E, Dikarev EV, Glass RS, Jin J, Li B, Li X, Zhang SZ (2006) Synthesis, structure, and chemistry of new, mixed group 14 and 16 heterocycles: nucleophile-induced ring contraction of mesocyclic dications. J Am Chem Soc 128:14949-14961

40. Kamper SG, Porter-Peden L, Blankespoor R, Sinniah K, Zhou D, Abell C, Rayment T (2007) Investigating the specific interactions between carbonic anhydrase and a sulfonamide inhibitor by singlemolecule force spectroscopy. Langmuir 23:12561-12565

41. Kim F, Song JH, Yang P (2002) Photochemical synthesis of gold nanorods. J Am Chem Soc 124:14316-14317

42. Nikoobakht B, El-Sayed MA (2001) Evidence for bilayer assembly of cationic surfactants on the surface of gold nanorods. Langmuir 17: 6368-6374

43. Placido T, Comparelli R, Giannici F, Cozzoli PD, Capitani G, Striccoli M, Agostiano A, Curri ML (2009) Photochemical synthesis of water-soluble gold nanorods: the role of silver in assisting anisotropic growth. Chem Mater 21:4192-4202

44. Bethencourt MI, Srisombat L, Chinwangso P, Randall Lee T (2009) SAMs on gold derived from the direct adsorption of alkanethioacetates are inferior to those derived from the direct adsorption of alkanethiols. Langmuir 25:1265-1271 
45. Cai LT, Yao YX, Yang JP, Price DW, Tour JM (2002) Chemical and potential-assisted assembly of thiolacetyl-terminated oligo(phenylene ethynylene)s on gold surfaces. Chem Mater 14:2905-2909

46. McFarland AD, Young MA, Dieringer JA, Van Duyne RP (2005) Wavelength-scanned surface-enhanced Raman excitation spectroscopy. J Phys Chem B 109:11279-11285

47. Draine BT, Flatau PJ (1994) Discrete-dipole approximation for scattering calculations. J Opt Soc Am A 113:1491-1499

48. Draine BT, Flatau PJ (2010) User guide for the discrete dipole approximation code DDSCAT version 7.1. http://arxiv.org/abs/1002.1505

49. González AL, Reyes-Esqueda JA, Noguez C (2008) Optical properties of elongated noble metal nanoparticles. J Phys Chem C 112: 7356-7362
50. Amendola V, Bakr OM, Stellacci F (2010) A study of the surface plasmon resonance of silver nanoparticles by the discrete dipole approximation method: effect of shape, size, structure, and assembly. Plasmonics 5:85-97

51. N'Gom M, Li SZ, Schatz G, Erni R, Agarwal A, Kotov N, Norris TB (2009) Electron-beam mapping of plasmon resonances in electromagnetically interacting gold nanorods. Phys Rev B 80:113411

52. Palik ED (1985) Handbook of optical constants of solids

53. Amendola V, Meneghetti M (2009) Size evaluation of gold nanoparticles by UV-Vis spectroscopy. J Phys Chem C 113:4277-4285

54. Kreibig U, Vollmer M (1995) Optical properties of metal clusters. Springer, Berlin 